\title{
Length at first sexual maturity of economically important fishes in the Brazilian Northeast Coast
}

\author{
Yago Bruno Silveira Nunes ${ }^{1 *}$ Mariana Barros Aranha $^{1}{ }^{\oplus}$, Jailza Freitas ${ }^{1}{ }^{\oplus}$, Jackellynne \\ Fernanda Farias Fernandes ${ }^{1} \oplus$, Ladilson Rodrigues Silva ${ }^{1}$, Marina Bezerra Figueiredo' ${ }^{1}$ \\ 1 Universidade Estadual do Maranhão - Departamento de Engenharia de Pesca (Cidade Universitária Paulo VI - CP 09 - Tirirical - 65055- \\ 970 - São Luís - MA - Brazil) \\ *Corresponding author: ybruno3098@gmail.com
}

Research involving fish stocks' reproductive aspects has social, economic, and biological importance; (Pinheiro, 2010; Véras and Almeida, 2016). Studies dealing with mean fish length at first sexual maturity are essential to assess and manage the exploited fish population and determine the minimum permissible size for capture, since some marine species have been overfished (King and McFarlane, 2003; Chellappa et al., 2010). According to Pinheiro (2010), the mean length at first sexual maturity represents the mean fish size when $50 \%$ of the individuals are ready to enter a new cohort, actively participating in the reproductive process.

This work's objective was to estimate the mean length at first sexual maturity of economically important fishes in the Brazilian Northeast coast, thus improving the biological understanding of these species in the light of more efficient stock management.

Samples from the following species were captured from 2013 to 2019, through artisanal fishery brought to the municipality of Raposa, in the state of Maranhão, Brazil (Figure 1): Genyatremus luteus (478 individuals - 182M; 296F), Micropogonias furnieri (190 individuals - 107M; 83F), Oligoplites palometa (172 individuals - 97M; 75F), Nebris microps (95 individuals

Submitted on: 10/February/2020

Approved on: 4/May/2020

Associate Editor: Francesc Maynou

Editor: Rubens M. Lopes

(C) (i) 2020 The authors. This is an open access article distributed under the terms of the Creative Commons license.
- 36M; 59F), Peprilus crenulatus (84 individuals - 43M; 41F), Trichiurus lepturus (146 individuals - 43M; 103F), Selene setapinnis (102 individuals - 62M; 40F), Bagre bagre (123 individuals - 55M; 68F), Lutjanus synagris (359 individuals - 149M; 210F) and Mugil curema (75 individuals - 29M; 46F).

The following data were collected: total (Lt), and furcal (Lf) lengths ( $\mathrm{mm})$, total (Wt), and eviscerated (We) weights (g). Lengths were measured using a ruler, and weights were determined with a balance with a precision of $0.01 \mathrm{~g}$. The fishes were longitudinally cut in the ventral part to remove the gonads, which were fixed in a $5 \%$ formalin solution for subsequent preservation in $70 \%$ ethanol.

The determination of young and adult specimens was done by verifying the gonad sexual maturity level according to the stages suggested by BrownPeterson et al. (2011) and Lowerre-Barbieri et al. (2011).

The mean fish length at first sexual maturity (L50) was estimated from the accumulated curve of the frequencies of occurrence of adult specimens per length class fitted to a logistic function. The fish maturation stages were grouped in young (stage $A$ ) and adult (stages $B, C$, and $D$ ). The immature stage $(A)$ was similar in both sexes characterized by the small size and light color of the gonads. The development stage (B) had a larger size than the previous one and by the macroscopic visualization of blood vessels and small oocytes. The spawning stage (C) presented larger-sized gonads with prominent blood vessels. When gonads were in the regressing stage (D), they had darker coloration and flaccidity. 


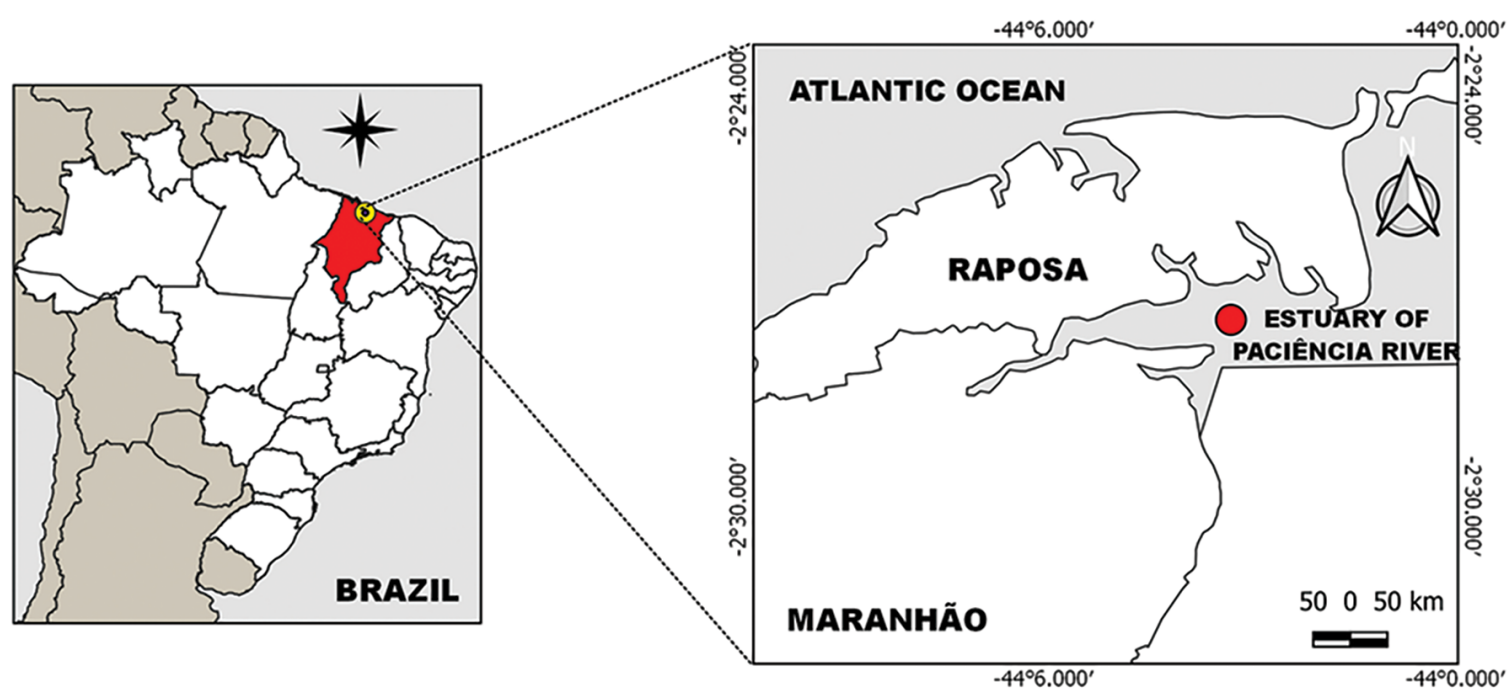

Figure 1. Location of the municipality of Raposa, in the state of Maranhão, Brazil.

The percentage of mature fish per length class was calculated and considered as dependent variable $(\mathrm{Y})$, and the total length as an independent variable (X). Subsequently, the values were fitted to a logistic curve, according to the formula:

$$
P=1 /(1+\exp [-r(L-L m)])
$$

Where $\mathrm{P}$ is the proportion of mature specimens, $\mathrm{r}$ is the curve slope, $L$ is the length, and $L m$ is the mean length at sexual maturity. The total length $(\mathrm{Lt})$ of the specimens evaluated was used as a morphometric measure for the calculations. Protocol 037/2018 of the Ethics Committee on animal use of the State University of Maranhão was respected.

When considering fishes from both sexes that are economically important in the North Coast of Maranhão, Brazil (Table 1), the L50 indicated that $4.9 \%$ of the $T$. lepturus specimens were immature when captured. The other species evaluated presented proportions of immature specimens of 26.3\% (Nebris microps), 34.5\% (P. crenulatus), 31.7\% (B. bagre), $11.6 \%$ (M. furnieri), $17.6 \%$ (S. setapinnis), $18.9 \%$ (O. palometa), 28\% (M. curema), $21.7 \%$ (G. luteus) and $12.2 \%$ (L. synagris).

The species O. palometa (Berry and Smith-Vaniz, 1978; Duque-Nivia et al., 1995), L. synagris (Freitas, 2009; Lessa et al., 2004; Trindade-Santos and Freire, 2015) and B. bagre (Véras and Almeida, 2016) had a higher L50 than that calculated in previous studies. The species M. furnieri (Carneiro et al., 2005; Haimovici and Ignacio, 2005; Santos et al., 2015), T. lepturus (Magro, 2005; CMFRI, 2016; Costa et al., 2018) and M. curema (Araújo and Silva, 2013; Trindade-Santos and Freire, 2015) had similar or lower L50 compared to other studies. S. setappinis showed significant variation when comparing its $L 50$ with other studies in Brazil (Bastos et al., 2005; Costa et al., 2018). N. microps, G. luteus, and P. crenulatus have no L50 information from previous studies.

The estimated L50 showed that most of the species presented maturing females, with sizes relatively larger than males (Table 2 ). However, $P$. crenulatus and $S$. setapinnis showed an opposite trend, probably because of the lack of selectivity of the fishing gears used in the captures. Based on the maturity size, management measures for these stocks should be considered by establishing a permissible capturing size. The number of specimens captured below the L50 was relatively low for all species evaluated, compared to mature fishes.

\section{ACKNOWLEDGMENTS}

We thank the support of the Fundação de Amparo à Pesquisa e ao Desenvolvimento Científico e Tecnológico do Maranhão (FAPEMA), as well as the State University of Maranhão (UEMA), and finally the Laboratory of Fisheries Biology (BIOPESQ). 
Table 1. Variation of total length and length at first sexual maturity $\left(\mathrm{L}_{50}\right)$, considering both sexes of economically important fishes in the North Coast of the state of Maranhão, Brazil.

\begin{tabular}{|c|c|c|c|c|c|c|c|}
\hline \multirow{2}{*}{ Order } & \multirow{2}{*}{ Family } & \multirow{2}{*}{ Species } & \multicolumn{3}{|c|}{ Total length $(\mathrm{cm})$} & \multirow{2}{*}{$\begin{array}{l}\text { Immature } \\
(\%)\end{array}$} & \multirow{2}{*}{$\begin{array}{l}\mathrm{L}_{50} \\
\text { considering } \\
\text { both sexes } \\
\text { (cm) }\end{array}$} \\
\hline & & & MinLt & MaxLt & $M \pm S D$ & & \\
\hline \multirow{8}{*}{ Perciformes } & Sciaenidae & $\begin{array}{l}\text { Nebris microps } \\
\text { Cuvier, } 1830\end{array}$ & 23.0 & 36.3 & $30.29 \pm 3.23$ & 26.3 & 23.33 \\
\hline & Sciaenidae & $\begin{array}{l}\text { Micropogonias furnieri } \\
\text { Desmarest, } 1823\end{array}$ & 30.6 & 46.5 & $37.84 \pm 3.41$ & 11.6 & 30.73 \\
\hline & Carangidae & $\begin{array}{l}\text { Oligoplites palometa } \\
\text { Cuvier, } 1832\end{array}$ & 29.4 & 54.7 & $38.59 \pm 4.78$ & 18.9 & 30.76 \\
\hline & Trichiuridae & $\begin{array}{l}\text { Trichiurus lepturus } \\
\text { Linnaeus, } 1758\end{array}$ & 55.5 & 111.0 & $78.36 \pm 7.06$ & 4.9 & 60.37 \\
\hline & Haemulidae & $\begin{array}{l}\text { Genyatremus luteus } \\
\text { Bloch, } 1790\end{array}$ & 12.0 & 33.0 & $23.18 \pm 3.67$ & 21.7 & 14.40 \\
\hline & Stromateidae & $\begin{array}{l}\text { Peprilus crenulatus } \\
\text { Cuvier, } 1829\end{array}$ & 10.9 & 22.0 & $14.91 \pm 2.12$ & 34.5 & 10.87 \\
\hline & Carangidae & $\begin{array}{c}\text { Selene setapinnis } \\
\text { Mitchill, } 1815\end{array}$ & 13.4 & 42.0 & $27.46 \pm 5.50$ & 17.6 & 24.00 \\
\hline & Lutjandae & $\begin{array}{l}\text { Lutjanus synagris } \\
\text { Linnaeus, } 1758\end{array}$ & 24.9 & 53.0 & $35.24 \pm 4.68$ & 12.2 & 26.13 \\
\hline Siluriformes & Ariidae & $\begin{array}{c}\text { Bagre bagre } \\
\text { Linnaeus, } 1766\end{array}$ & 21.0 & 51.0 & $31.87 \pm 6.16$ & 31.7 & 24.16 \\
\hline Mugiliformes & Mugilidae & $\begin{array}{c}\text { Mugil curema } \\
\text { Valenciennes, } 1836\end{array}$ & 12.0 & 30.0 & $20.31 \pm 3.45$ & 28.0 & 16.49 \\
\hline
\end{tabular}

MinLt $=$ Minimum total length; MaxLt $=$ Maximum total length; $M \pm S D=$ Mean \pm Standard deviation .

Table 2. Minimum and maximum length, at first sexual maturity $\left(\mathrm{L}_{50}\right)$ for male and female of commercially important fish in the North Coast of the state of Maranhão, Brazil.

\begin{tabular}{|c|c|c|c|c|c|c|c|c|}
\hline \multirow[b]{2}{*}{ Species } & \multicolumn{4}{|c|}{ Male } & \multicolumn{4}{|c|}{ Female } \\
\hline & MinLt & MaxLt & Maturity equation & $\begin{array}{c}\text { Immature } \\
(\%)\end{array}$ & MinLt & MaxLt & Maturity equation & $\begin{array}{c}\text { Immature } \\
(\%)\end{array}$ \\
\hline Nebris microps & 23.0 & 35.4 & $\begin{array}{c}y=1 /(1+\exp (- \\
\left.\left.(0.13)^{*}(x-(26.04))\right)\right)\end{array}$ & 44.4 & 24.5 & 36.3 & $\begin{array}{c}y=1 /(1+\exp (- \\
\left.\left.(1.64)^{*}(x-(25.41))\right)\right)\end{array}$ & 15.5 \\
\hline $\begin{array}{l}\text { Micropogonias } \\
\text { furnieri }\end{array}$ & 30.6 & 46.5 & $\begin{array}{c}y=1 /(1+\exp (- \\
\left.\left.(0.26)^{*}(x-(32.97))\right)\right)\end{array}$ & 7.7 & 32.4 & 42.4 & $\begin{array}{c}y=1 /(1+\exp (- \\
\left.\left.(0.51)^{*}(x-(32.36))\right)\right)\end{array}$ & 16.1 \\
\hline $\begin{array}{l}\text { Oligoplites } \\
\text { palometa }\end{array}$ & 29.4 & 52.2 & $\begin{array}{c}y=1 /(1+\exp (- \\
\left.\left.(0.52)^{*}(x-(29.91))\right)\right)\end{array}$ & 11.8 & 33.2 & 54.7 & $\begin{array}{c}y=1 /(1+\exp (- \\
\left.\left.(0.18)^{*}(x-(32.32))\right)\right)\end{array}$ & 26.4 \\
\hline Trichiurus lepturus & 61.0 & 94.0 & $\begin{array}{c}y=1 /(1+\exp (- \\
\left.\left.(0.02)^{*}(x-(60.25))\right)\right)\end{array}$ & 9.3 & 55.5 & 111 & $\begin{array}{c}\mathrm{y}=1 /(1+\exp (- \\
\left.\left.(1.24)^{*}(\mathrm{x}-(67.51))\right)\right)\end{array}$ & 9.7 \\
\hline $\begin{array}{l}\text { Genyatremus } \\
\text { luteus }\end{array}$ & 13.0 & 33.0 & $\begin{array}{c}y=1 /(1+\exp (- \\
\left.\left.(2.20)^{*}(x-(14.43))\right)\right)\end{array}$ & 6.5 & 12.0 & 33.0 & $\begin{array}{c}y=1 /(1+\exp (- \\
\left.\left.(0.18)^{*}(x-(16.90))\right)\right)\end{array}$ & 30.7 \\
\hline $\begin{array}{l}\text { Peprilus } \\
\text { crenulatus }\end{array}$ & 10.9 & 22.0 & $\begin{array}{c}y=1 /(1+\exp (- \\
\left.\left.(0.17)^{*}(x-(16.24))\right)\right)\end{array}$ & 51.1 & 11.0 & 20.5 & $\begin{array}{c}y=1 /(1+\exp (- \\
\left.\left.(3.07)^{*}(x-(11.48))\right)\right)\end{array}$ & 21.9 \\
\hline Selene setapinnis & 13.4 & 42.0 & $\begin{array}{c}y=1 /(1+\exp (- \\
\left.\left.(2.97)^{*}(x-(24.00))\right)\right)\end{array}$ & 16.1 & 13.7 & 33.5 & $\begin{array}{c}\mathrm{y}=1 /(1+\exp (- \\
\left.\left.(1.42)^{*}(\mathrm{x}-(22.03))\right)\right)\end{array}$ & 20 \\
\hline Lutjanus synagris & 24.9 & 44.0 & $\begin{array}{c}y=1 /(1+\exp (- \\
\left.\left.(1.08)^{*}(x-(26.00))\right)\right)\end{array}$ & 9.5 & 25.0 & 53.0 & $\begin{array}{c}y=1 /(1+\exp (- \\
\left.\left.(0.70)^{*}(x-(26.27))\right)\right)\end{array}$ & 16.1 \\
\hline Bagre bagre & 22.5 & 51.0 & $\begin{array}{c}y=1 /(1+\exp (- \\
\left.\left.(0.02)^{*}(x-(23.57))\right)\right)\end{array}$ & 44.2 & 21.0 & 51.0 & $\begin{array}{c}y=1 /(1+\exp (- \\
\left.\left.(0.31)^{*}(x-(24.61))\right)\right)\end{array}$ & 22.1 \\
\hline Mugil curema & 15.0 & 30.0 & $\begin{array}{c}y=1 /(1+\exp (- \\
\left.\left.(0.49)^{*}(x-(16.07))\right)\right)\end{array}$ & 27.5 & 12.0 & 29.5 & $\begin{array}{c}y=1 /(1+\exp (- \\
\left.\left.(0.91)^{*}(x-(17.03))\right)\right)\end{array}$ & 28.2 \\
\hline
\end{tabular}




\section{AUTHOR CONTRIBUTIONS}

Y. B. S. N.: Formal analysis; Writing - original draft.

M. B. A.: Data curation; Formal analysis.

J. F.: Data curation; Formal analysis.

J. F. F. F.: Data curation; Formal analysis.

L. R. S.: Data curation; Formal analysis.

M. B. F. Writing - review \& editing; Validation; Visualization.

\section{REFERENCES}

ARAÚJO, A. R. \& SILVA, F. D. 2013. Aspectos da pesca e biologia da tainha, Mugil curema (Osteichthyes: Mugilidae), no Estuário do Rio Vaza Barris, Sergipe, Brasil. Arquivos de Ciências do Mar, 46(1), 29-38. DOI: https://doi.org/10.32360/acmar.v46i1.888

BASTOS, C. M. L. F., CERGOLE, M. C., MAGRO, M., BASTOS, G. C. C. \& TREVIZAN, F. 2005. Selene setapinnis (Mitchell, 1815). In: CERGOLE, M. C., ÁVILA-DA-SILVA, A. O. \& ROSSI-WONGTCHOWSKI, C. L. D. B. (eds.). Análise das principais pescarias comerciais da região sudeste-sul do Brasil: dinâmica populacional das espécies em explotação. São Paulo: Instituto Oceanográfico.

BERRY, F. H. \& SMITH-VANIZ, W. F. 1978. Carangidae. In: FISCHER, W. (ed.). FAO species identification sheets for fishery purposes. Volume 1, West Atlantic (Fishing Area 31): FAO, Rome.

BROWN-PETERSON, N. J., WYANSKI, D. M., SABORIDOREY, F., MACEWICZ, B. J. \& LOWERRE-BARBIERI, S. K. 2011. A standardized terminology for describing reproductive development in fishes. Marine and Coastal Fisheries Dynamics, Management, and Ecosystem Science, 3(1), 52-70. DOI: https://doi.or $\mathrm{g} / 10.1080 / 19425120.2011 .555724$

CARNEIRO, M. H., CASTRO, P. M. G., TUTUI, S. L. S. \& BASTOS, G. C. C. 2005. Micropogonias furnieri (Desmarest, 1823). In: CERGOLE, M. C., ÁVILA-DASILVA, A. O. \& ROSSI-WONGTCHOWSKI, C. L. D. B. (eds.). Análise das principais pescarias comerciais da região sudeste-sul do Brasil: dinâmica populacional das espécies em explotação. São Paulo: Instituto Oceanográfico.
COSTA, M. R., TUBINO, R. A. \& MONTEIRO-NETO,

C. 2018. Length-based estimates of growth parameters and mortality rates of fish populations from a coastal zone in the Southeastern Brazil. Zoologia (Curitiba), 35, 1-8. DOI: https://doi. org/10.3897/zoologia.35.e22235

CHELLAPPA, S., LIMA, J. T. A. X., ARAÚJO, A. \& CHELLAPPA, N. T. 2010. Ovarian development and spawning of Serra Spanish mackerel in coastal waters of Northeastern Brazil. Brazilian Journal of Biology, 70(2), 631-637. DOI: http://dx.doi. org/10.1590/S1519-69842010005000012

CMFRI (Central Marine Fisheries Research Institute). 2016. CMFRI Annual report 2016-2017. Technical report. Kochi: Central Marine Fisheries Research Institute.

DUQUE-NIVIA， G., ARTHURO, A. P. \& SANTOSMARTINEZ, A. 1995. Aspectos reproductivos de Oligoplites saurus y 0 . palometa (Pisces: Carangidae) en La Ciénaga Grande de Santa Marta, Caribe Colombiam. Caribean Journal of Science, 31(3-4), 317-326.

FREITAS, M. O. 2009. Pesca artesanal e biologia reprodutiva do ariocó Lutjanus synagris (Lutjanidae) no Banco dos Abrolhos. M.e. Universidade Estadual de Santa Cruz. Available at: http://nbcgib.uesc.br/ppgsat/files/PPGSAT/ Dissertacoes/Matheus_Oliveira_Freitas.pdf [Accessed 05 Feb. 2020].

HAIMOVICl, M. \& IGNACIO, J. M. 2005. Micropogonias furnieri, (Desmarest, 1823). In: CERGOLE, M. C., ÁVILA-DA-SILVA, A. O. \& ROSSIWONGTCHOWSKI, C. L. D. B. (eds.). Análise das principais pescarias comerciais da região sudeste-sul do Brasil: dinâmica populacional das espécies em explotação. São Paulo: Instituto Oceanográfico.

KING, J. R. \& MCFARLANE, G. A. 2003. Marine fish life history strategies: applications to fishery management. Fisheries Management and Ecology, $10,249-264$. 
LESSA, R. P., NÓBREGA, M. F. \& BEZERRA JUNIOR, J. L. 2004. Dinâmica de populações e avaliação de estoques dos recursos pesqueiros da região nordeste. Volume II. Recife, Brazil: DIMAR, Departamento de Pesca Universidade Federal Rural de Pernambuco.

LOWERRE-BARBIERI, S. K., GANIAS, K., SABORIDO-REY, F., MURUA, H. \& HUNTER, J. R. 2011. Reproductive timing in marine fishes: variability, temporal scales, and methods. Marine and Coastal Fisheries:Dynamics, Management, and Ecosystem Science, 3(1), 71-91. DOI: https://doi.org/10.1080/19425120.2011.556932

MAGRO, M. 2005. Trichiurus lepturus Linnaeus, 1758. In: CERGOLE, M. C., ÁVILA-DA-SILVA, A. O. \& ROSSIWONGTCHOWSKI, C. L. D. B. (eds.). Análise das principais pescarias comerciais da região sudeste-sul do Brasil: dinâmica populacional das espécies em explotação. São Paulo: Instituto Oceanográfico.
PINHEIRO, M. S. S. 2010. Ciclo de vida e estrutura de uma assembléia de peixes teleósteos em um manguezal da Raposa, Maranhão, Brasil. Dr. Universidade Estadual Paulista. Available at: http://hdl.handle. net/11449/106603 [Accessed 05 Feb. 2020].

SANTOS, R. S., SILVA, J. P. C., COSTA, M. R. \& ARAÚJO, F. G. 2015. O tamanho de primeira maturação como parâmetro para estabelecimento de tamanho mínimo de captura para corvina no Sudeste do Brasil. Boletim do Instituto de Pesca, 41(3), 507-518.

TRINDADE-SANTOS, I. \& FREIRE, K. M. F. 2015. Analysis of reproductive patterns of fishes from three large marine ecosystems. Frontiers in Marine Science, 38, 1-10. DOI: https://doi.org/10.3389/fmars.2015.00038

VÉRAS, P. F. \& ALMEIDA, Z. S. 2016. Biologia reprodutiva do Bagre bagre capturado pela pescaria de zangaria. Revista Brasileira de Ciências Agrárias, 11(4), 367-373. DOI: https://doi.org/10.5039/agraria.v11i4a5402 